\title{
Quantum-memory-assisted entropic uncertainty relation with a single nitrogen-vacancy center in diamond
}

\author{
Z. Y. Xu ${ }^{1}$, 1, a) S. Q. Zhu ${ }^{1}$, 1, b) and W. L. Yang ${ }^{21}$ \\ ${ }^{1}$ School of Physical Science and Technology, Soochow University, Suzhou, 215006, China \\ ${ }^{2}$ State Key Laboratory of Magnetic Resonance and Atomic and Molecular Physics, Wuhan Institute of Physics and Mathematics, \\ Chinese Academy of Sciences, Wuhan, 430071, China
}

The limitation of simultaneous measurements of noncommuting observables can be eliminated when the measured particle is maximally entangled with a quantum memory. We present a proposal for testing this quantum-memory-assisted entropic uncertainty relation in a single nitrogen-vacancy $(\mathrm{N}-\mathrm{V})$ center in diamond only by local electronic measurements. As an application, this entropic uncertainty relation is used to witness entanglement between the electron and nuclear spins of the $\mathrm{N}-\mathrm{V}$ center, which is close to reach the currently available technology.

PACS numbers: 76.30.Mi, 42.50.Dv, 03.67.Bg, 03.67.Mn

The Heisenberg's uncertainty principle is the cornerstone of quantum mechanics, which provides a limitation of simultaneous measurements of canonically conjugate observables ${ }^{1}$. In quantum information theory, the uncertainty relation is usually characterized by entropic measures rather than standard deviations ${ }^{2,3}$. However, the traditional entropic uncertainty relation may be violated if particle $A$ to be measured is initially entangled with a quantum memory $B^{4}$. Recently, a quantum-memory-assisted entropic uncertainty relation has been conjectured ${ }^{5}$ and later proved with an equivalent form ${ }^{6}$

$$
S(Q \mid B)+S(R \mid B) \geq \log _{2} \frac{1}{c}+S(A \mid B) .
$$

Here, $S(\Lambda \mid B)$ with $\Lambda \in(Q, R)$ is the conditional von Neumann entropy of the post-measurement state $\rho_{\Lambda B}=$ $\sum_{m}\left(\left|\psi_{m}\right\rangle\left\langle\psi_{m}\right| \otimes \mathbb{1}\right) \rho_{A B}\left(\left|\psi_{m}\right\rangle\left\langle\psi_{m}\right| \otimes \mathbb{1}\right)$, where $\mathbb{1}$ is the identity operator and $\left\{\left|\psi_{m}\right\rangle\right\}$ are the eigenstates of the observable $\Lambda$. The parameter $\log _{2} \frac{1}{c}$ quantifies the incompatibility of two measurements with $c=\max _{\alpha, \beta}\left|\left\langle\phi_{\alpha} \mid \varphi_{\beta}\right\rangle\right|^{2}$, where $\left|\phi_{\alpha}\right\rangle$ and $\left|\varphi_{\beta}\right\rangle$ are the eigenstates of two noncommuting observables $Q$ and $R$, respectively. Equation (1) can be understood in a straightforward way: the entanglement between $A$ and $B$ may create a negative conditional entropy $S(A \mid B)^{7}$, which will beat the incompatibility $\log _{2} \frac{1}{c}$. In other words, the quantum information held by the quantum memory $B$ will help us to eliminate the uncertainty of the measurements performed on particle $A$. This entropic uncertainty relation together with other forms ${ }^{8-10}$ has practical applications such as for witnessing entanglement ${ }^{6}$ and cryptography ${ }^{11}$ and has been explored under noise $^{12}$ and in optical photon systems ${ }^{13,14}$.

Over the past few years, the negatively charged nitrogenvacancy $(\mathrm{N}-\mathrm{V})$ center in diamond has been considered as one of the most promising building block for solid-state quantum information processing ${ }^{15}$. Indeed, due to weak magnetic interaction with the environment, the proximal nuclear spins in a single $\mathrm{N}-\mathrm{V}$ center become a good quantum memory for information storage ${ }^{16-18}$. In addition, the paramagnetic electron spin in a single $\mathrm{N}-\mathrm{V}$ center can be optically polarized and

\footnotetext{
a)Electronic mail: zhenyuxu@ suda.edu.cn

b)Electronic mail: szhu@suda.edu.cn
}

readout with high fidelity at room temperature ${ }^{19}$ and can also be used to polarize and readout the nuclear spins $\mathrm{s}^{20}$ and realize conditional gating ${ }^{21,22}$. Based on above advantages, the $\mathrm{N}-\mathrm{V}$ center provides an excellent test bed to explore this quantummemory-assisted entropic uncertainty relation.

In this Letter, we present a practical proposal to verify this entropic uncertainty relation in a single $\mathrm{N}-\mathrm{V}$ center in diamond by local electronic measurements. As a by-product, this entropic uncertainty relation is employed to witness entanglement of electron and nuclear spins in diamond. The experimental feasibility is also justified with current laboratory parameters.

Before introducing the experimental proposal, we first display a theoretical framework of the quantum-memoryassisted entropic uncertainty relation for two-qubit system, in which an arbitrary two-qubit state can be of the form ${ }^{23}$ $\rho_{A B}=\frac{1}{4}\left[\mathbb{1}^{A} \otimes \mathbb{1}^{B}+\sum_{i=1}^{3}\left(x_{i} \sigma_{i}^{A} \otimes \mathbb{1}^{B}+\mathbb{1}^{A} \otimes y_{i} \sigma_{i}^{B}\right)+\right.$ $\left.\sum_{i, j=1}^{3} T_{i j} \sigma_{i}^{A} \otimes \sigma_{j}^{B}\right]$, where $\sigma_{i(j)}$ with $i(j) \in\{1,2,3\}$ correspond to standard Pauli matrices. We define two $3 \times 1$ vectors $\mathbf{x}$ with real components $x_{i}=\operatorname{tr}_{A B}\left(\rho_{A B} \sigma_{i}^{A} \otimes \mathbb{1}^{B}\right)$ and $\mathbf{y}$ with real components $y_{i}=\operatorname{tr}_{A B}\left(\rho_{A B} \mathbb{1}^{A} \otimes \sigma_{i}^{B}\right)$, and a $3 \times 3$ correlation tensor $\overline{\mathbf{T}}$ with real components $T_{i j}=\operatorname{tr}_{A B}\left(\rho_{A B} \sigma_{i}^{A} \otimes \sigma_{j}^{B}\right)$. If we choose two Pauli observables $Q=\sigma_{1}$ and $R=\sigma_{3}$ to be measured, the uncertainty, i.e., the left hand side of Eq. (1), can be expressed as

$$
U=-\sum_{\substack{\mu, \nu=0,1 \\ \lambda=1,3}} \eta_{\mu \nu}^{\lambda} \log _{2}\left(\eta_{\mu \nu}^{\lambda}\right)-2 H_{b i n}\left(\frac{1-\|\mathbf{y}\|}{2}\right)
$$

where $H_{b i n}(p)=-p \log _{2} p-(1-p) \log _{2}(1-p)$ denotes the binary entropy ${ }^{7}, \eta_{\mu \nu}^{\lambda}=\left[1+(-1)^{\mu} x_{\lambda}+\right.$ $\left.(-1)^{\nu} \sqrt{\sum_{i=1}^{3}\left(y_{i}+(-1)^{\mu} T_{\lambda i}\right)^{2}}\right] / 4$, and $\|\mathbf{y}\|=\sqrt{\sum_{i=1}^{3} y_{i}^{2}}$. Since the complementarity $c$ of the observables $\sigma_{1}$ and $\sigma_{3}$ is always equal to $1 / 2$, the lower bound of uncertainty, i.e., the right hand side of Eq. (1), takes the form

$$
U_{b}=S\left(\rho_{A B}\right)+1-H_{b i n}\left(\frac{1-\|\mathbf{y}\|}{2}\right) .
$$

In experiment, if we choose the same measurement $\Lambda$ on particles $A$ and $B$, we may get $H(\Lambda \mid \Lambda) \geq S(\Lambda \mid B)$ with $H(\cdot)$ 

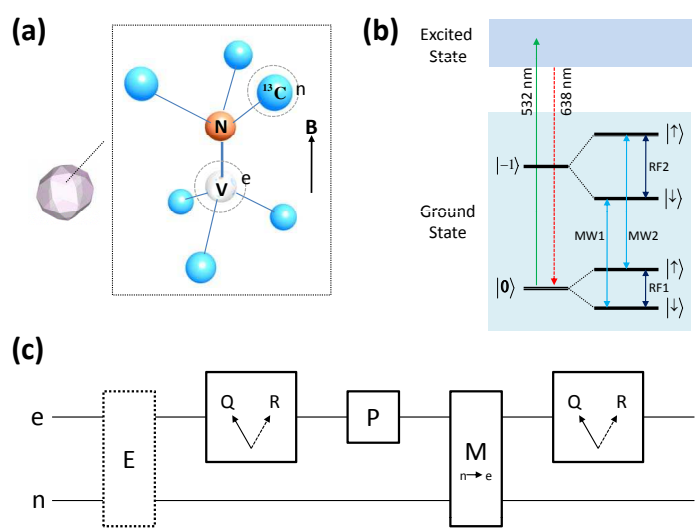

FIG. 1. (Color online) (a) Structure of a single N-V center in diamond including the electron spin and the ${ }^{13} \mathrm{C}$ nuclear spin. An external magnetic field $\mathbf{B}$ is applied along the [111] axis of the N-V center to form the quantization axis. (b) Electron-nuclear spin level diagram, where electronic ground states $\{|0\rangle,|-1\rangle\}$ and nuclear spin states $\{|\downarrow\rangle,|\uparrow\rangle\}$ are employed to encode the two-qubit system. (c) Schematics for testing the quantum-memory-assisted entropic uncertainty relation and witnessing the entanglement between electron and nuclear spins. The first dashed box is only for testing the uncertainty relation, which is not necessary for the procedures of witnessing entanglemant. $Q=\sigma_{1}$ and $R=\sigma_{3}$ in the second box are two noncommuting observables. The third box represents the polarization of electron spin and the forth box is used to transfer the nuclear state to the electronic state by mapping operations, which will be followed by the fifth box by performing the same measurement as the second box on the electron spin again.

the Shannon entropy. According to Fano's inequality ${ }^{7}$, we have $H(\Lambda \mid \Lambda) \leq H_{\text {bin }}\left(\kappa_{\Lambda}\right)$ with $\kappa_{\Lambda}$ the probability that the outcomes of the same measurement $\Lambda$ on particles $A$ and $B$ are different. Therefore, $H_{b i n}\left(\kappa_{\Lambda}\right) \geq S(\Lambda \mid B)$, which will in general lead to a higher measurement estimation $(M E)$ of the uncertainty, and can be used to conveniently test $U$ with experimental counts ${ }^{13,14}$. For two-qubit states and observables $\sigma_{1}$ and $\sigma_{3}$,

$$
M E=H_{b i n}\left(\frac{1-T_{11}}{2}\right)+H_{b i n}\left(\frac{1-T_{33}}{2}\right) .
$$

In Fig. 1, the structure of a pure N-V center in diamond is depicted, where we treat the electron $\operatorname{spin}(S=1)$ as particle $A$ and a proximal ${ }^{13} \mathrm{C}$ nuclear spin $(I=1 / 2)$ as quantum memory $B$. In the following, we will employ the electronic states $\{|0\rangle,|-1\rangle\}$ and the nuclear states $\{|\downarrow\rangle,|\uparrow\rangle\}$ as two-qubit system [Fig. 1(b)].

An experimental schematic sequence for testing this quantum-memory-assisted entropic uncertainty relation is depicted in Fig. 1(c). The first dashed box represents the entangled state (for example, we consider the Schmidt state $|\Phi\rangle=\cos \chi|0 \downarrow\rangle+\sin \chi|-1 \uparrow\rangle$ with $\chi \in[0, \pi / 2])$ preparation with the following key steps ${ }^{16}$ : (i) The electronic ground state is first polarized at state $|0\rangle$ by a $532 \mathrm{~nm}$ green laser pulse and the two-qubit state now reads $|0\rangle\langle 0| \otimes \rho_{n}$ with $\rho_{n}$ an unknown nuclear mixed state; (ii) Then we can transfer the nuclear state to the electronic state $|0\rangle\langle 0| \otimes \rho_{n} \rightarrow$ (a)

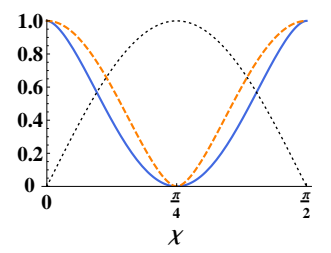

(b)

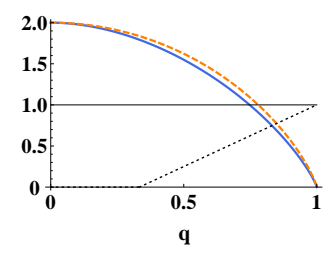

FIG. 2. (Color online) (a) An example of testing the quantummemory-assisted entropic uncertainty relation with Schmidt state $|\Phi\rangle=\cos \chi|0 \downarrow\rangle+\sin \chi|-1 \uparrow\rangle$ and observables $Q=\sigma_{1}$ and $R=\sigma_{3}$. The uncertainty $\left(U=U_{b}\right)$, measurement estimation $(M E)$, and the concurrence $(C)$ are represented by blue solid, orange dashed, and black dotted curves respectively. (b) Entanglement witness of electron and nuclear spins in a single $\mathrm{N}-\mathrm{V}$ center. The blue solid, orange dashed, and black dotted curves denotes $U_{b}, U=M E$, and $C$, respectively.

$\rho_{e} \otimes|\downarrow\rangle\langle\downarrow|$ with a conditional MW1- $\pi$ pulse followed by a conditional RF1- $\pi$ pulse; (iii) The electronic state can then be polarized again by the $532 \mathrm{~nm}$ laser pulse, which reduces the two-qubit state to $|0\rangle|\downarrow\rangle$; (iv) A MW1-2 $\chi$ pulse is then performed on the electronic state, which yields the product state $(\cos \chi|0\rangle+\sin \chi|-1\rangle) \otimes|\downarrow\rangle$. Then a conditional RF2- $\pi$ pulse will create the final entangled state $|\Phi\rangle$.

After preparation of the entangled state, we then perform measurement $Q=\sigma_{1}$ (or $R=\sigma_{3}$ ) on the electron spin. In our case, the $\sigma_{3}$ operation can be directly manipulated by electron shelving projecting onto $|0\rangle$ or $|-1\rangle$ with $532 \mathrm{~nm}$ laser pulse, and the $\sigma_{1}$ operation can be reduced to detecting $\sigma_{3}$ by applying a stronger MW- $\pi / 2$ pulse before and after $\sigma_{3}$ operation (i.e., $\sigma_{1}=\mathcal{H} \sigma_{3} \mathcal{H}$ with $\mathcal{H}$ the Hadamard gate).

To acquire the measurement estimation of the uncertainty in experiment, the same observable $Q=\sigma_{1}\left(\right.$ or $\left.R=\sigma_{3}\right)$ should be performed on the proximal ${ }^{13} C$ nuclear spin. However, since electron spin is good for efficient processing and readout, while the nuclear spin is more suitable for long-term storage $^{24}$, we will transfer the nuclear state to the electron spin and then perform the same operation $\sigma_{1}\left(\right.$ or $\left.\sigma_{3}\right)$ on the electron spin to quantify the uncertainty. This task can be accomplished by first polarizing the electron spin to the state $|0\rangle$ again [the 3rd box in Fig. 1(c)], and then employing the conditional MW2- $\pi$ pulse followed by a conditional RF2- $\pi$ pulse to transfer the nuclear state to the electronic state [the 4th box in Fig. 1(c)]. Finally, we may perform the $\sigma_{1}$ (or $\sigma_{3}$ ) operation on the electron spin again [the 5th box in Fig. 1(c)]. In comparison with the first and the second outcomes of $\sigma_{1}$ (or $\left.\sigma_{3}\right)$ operation [the 2nd and 5th boxes in Fig. 1(c)], we may acquire the measurement estimation of the uncertainty.

For state $|\Phi\rangle$ with $\mathbf{x}=\mathbf{y}=(0,0, \cos 2 \chi)^{t} \quad$ and $\overline{\mathbf{T}}=\operatorname{diag}\{\sin 2 \chi,-\sin 2 \chi, 1\}$, it is easy to check that the uncertainty $U=U_{b}=1-H_{b i n}\left(\sin ^{2} \chi\right)$ and the measurement estimation $M E=H_{b i n}\left(\frac{1-\sin 2 \chi}{2}\right)$. In Fig. 2(a), the dependence of the uncertainty $U$ and the measurement estimation $M E$ on the Schmidt state angle $\chi$ is plotted. The blue solid line represents the uncertainty $U$ and the lower bound $U_{b}$ (the equality is achieved in this case). The 


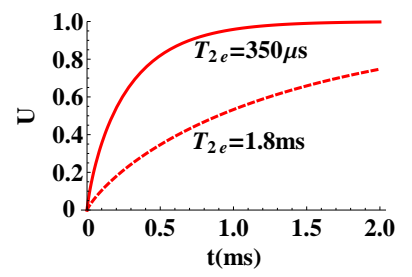

FIG. 3. (Color online) Electronic dephasing effect on the uncertainty $U\left(=U_{b}=M E\right)$ of observables $Q=\sigma_{1}$ and $R=\sigma_{3}$ performed on the state $\left|\Phi^{+}\right\rangle=\frac{1}{\sqrt{2}}(|0 \downarrow\rangle+|-1 \uparrow\rangle)$. Parameters are adopted in Ref. ${ }^{27}$ with $T_{2 e}=350 \mu \mathrm{s}$ and in Ref. ${ }^{28}$ with $T_{2 e}=1.8 \mathrm{~ms}$.

orange dashed line represents the measurement estimation, which is higher than the uncertainty. To illustrate the role of entanglement on this quantum-memory-assisted entropic uncertainty relation, we employ the concurrence ${ }^{25}$ with $C=2 \max \{0,|\sin \chi \cos \chi|\}$ [black dotted line in Fig. 2(a)] to characterize the entanglement. It is clearly illustrated that when the electron and nuclear spins are in a maximum entangled state $(\chi=\pi / 4)$, the uncertainty of two noncommuting observables can be totally eliminated.

One of the most remarkable applications of this quantummemory-assisted entropic uncertainty relation is to witness entanglement of electron and nuclear spins in the N-V center. Our scheme with only local electronic measurements and no need for quantum state tomography is helpful for experimental implementation. The procedures are similar to the former testing case except for the initial state preparation, i.e., the preparation of initial entangled state is actually not required, so the first box in Fig. 1(c) could be removed. However, to illustrate the application on the entanglement witness, we assume that the electron and nuclear spins are in state $|\Xi\rangle=\frac{1-q}{4} \mathbb{1} \otimes \mathbb{1}+q\left|\Phi^{+}\right\rangle\left\langle\Phi^{+}\right|$, with $\left|\Phi^{+}\right\rangle=$ $\frac{1}{\sqrt{2}}|0 \downarrow\rangle+|-1 \uparrow\rangle$. For state $|\Xi\rangle$ with $\mathbf{x}=\mathbf{y}=(0,0,0)^{t}$ and $\overline{\mathbf{T}}=\operatorname{diag}\{q,-q, q\}$, we have $U=M E=2 H_{b i n}\left(\frac{1-q}{2}\right)$, $U_{b}=H_{b i n}\left(\frac{1+3 q}{4}\right)+\frac{3(1-q)}{4} \log _{2} 3$, and $C=2 \max \left\{0, \frac{3 q-1}{4}\right\}$. As shown in Fig. 2(b), the electron and nuclear spins must be entangled if the uncertainty is less than 1.

Finally, we survey the relevant experimental parameters. As reported in recent $\mathrm{N}-\mathrm{V}$ experiments, the longitudinal relaxation time $T_{1 n}$ of ${ }^{13} \mathrm{C}$ nuclear spin is around $1.7 \mathrm{~s}^{18}$, and the coherence time $T_{2 n}$ is about $20 \mathrm{~ms}^{16}$ (We note that $T_{2 n}$ of ${ }^{13} \mathrm{C}$ can reach on the order of one second with elegant dissipative decoupling technique (DDT) ${ }^{18}$. However, the straight forward employment of DDT in our scheme will also induce complications with ionization and deionization of the $\mathrm{N}-\mathrm{V}$ center). While for $\mathrm{N}-\mathrm{V}$ electron spin at room temperature, the relaxation time $T_{1 e}=6 \mathrm{~ms}$ is reported in Ref. ${ }^{26}$ and coherence time $T_{2 e}$ ranges from from $350 \mu s^{27}$ to $1.8 \mathrm{~ms}^{28}$. Since the key step of our proposal is the state mapping from nucleus to electron, which will take on the time scale of 100 $\mu s^{22}, T_{2 e}$ becomes the key factor in our proposal $\left(T_{2 n}=20\right.$ $m s^{16}$ is long enough for implementing our proposal). Here, we briefly study the electronic dephasing effect on this en- tropic uncertainty relation. As an example, the initial state $\left|\Phi^{+}\right\rangle=\frac{1}{\sqrt{2}}(|0 \downarrow\rangle+|-1 \uparrow\rangle)$ under electronic dephasing effect yields $U=U_{b}=M E=H_{b i n}\left(\frac{1-e^{-\frac{t}{2 T_{2 e}}}}{2}\right)$, which is depicted in Fig. 3. Clearly, longer electronic dephasing time $T_{2 e}$ will retain less uncertainty of two incompatible measurements.

In conclusion, the quantum-memory-assisted entropic uncertainty relation has been explored in a single $\mathrm{N}-\mathrm{V}$ center in diamond. By investigating relevant experimental parameters, our proposal can be immediately verified under current $\mathrm{N}-\mathrm{V}$ based experimental conditions.

This work is supported by NNSFC under Grant Nos. 11204196, 11074184, 11004226, and 11274351.

${ }^{1}$ W. Heisenberg, Z. Phys. 43, 172 (1927).

${ }^{2}$ I. Bialynicki-Birula and Ł. Rudnicki, Statistical Complexity: Applications in Electronic Structure-Chapter One (Springer, 2011).

${ }^{3}$ S. Wehner and A. Winter, New J. Phys. 12, 025009 (2010).

${ }^{4}$ Y.-K. Kim and Y. Shih, Found. Phys. 29, 1849 (1999).

${ }^{5}$ J. M. Renes and J.-C. Boileau, Phys. Rev. Lett. 103, 020402 (2009).

${ }^{6}$ M. Berta, M. Christandl, R. Colbeck, J. M. Renes, and R. Renner, Nature Phys. 6, 659 (2010).

${ }^{7}$ M. A. Nielsen and I. L. Chuang, Quantum Computation and Quantum Information (Cambridge University Press, Cambridge, England, 2000).

${ }^{8}$ M. Tomamichel and R. Renner, Phys. Rev. Lett. 106, 110506 (2011).

${ }^{9}$ P. J. Coles, R. Colbeck, L. Yu, and M. Zwolak, Phys. Rev. Lett. 108, 210405 (2012).

${ }^{10}$ R. L. Frank and E. H. Lieb, e-print arXiv:1204.0825.

${ }^{11}$ M. Tomamichel, C. C. W. Lim, N. Gisin, and R. Renner, Nature Commun. 3, 634 (2012).

${ }^{12}$ Z. Y. Xu, W. L. Yang, and M. Feng, Phys. Rev. A 86, 012113 (2012).

${ }^{13}$ C.-F. Li, J.-S. Xu, X.-Y. Xu, K. Li, and G.-C. Guo, Nature Phys. 7, 752 (2011).

${ }^{14}$ R. Prevedel, D. R. Hamel, R. Colbeck, K. Fisher, and K. J. Resch, Nature Phys. 7, 757 (2011)

${ }^{15}$ J. R. Weber, W. F. Koehl, J. B. Varley, A. Janotti, B. B. Buckley, C. G. Van de Walle and D. D. Awschalom, PNAS 107, 8513 (2010).

${ }^{16}$ M. V. Gurudev Dutt, L. Childress, L. Jiang, E. Togan, J. Maze, F. Jelezko, A. S. Zibrov, P. R. Hemmer and M. D. Lukin, Science 316, 1312 (2007).

${ }^{17}$ G. D. Fuchs, G. Burkard, P. V. Klimov, and D. D. Awschalom, Nature Phys. 7, 789 (2011).

${ }^{18}$ P. C. Maurer, G. Kucsko, C. Latta, L. Jiang, N. Y. Yao, S. D. Bennett, F. Pastawski, D. Hunger, N. Chisholm, M. Markham, D. J. Twitchen, J. I. Cirac, M. D. Lukin, Science 336, 1283 (2012).

${ }^{19}$ N. B. Manson, L. J. Rogers, R. L. McMurtrie, M. W. Doherty, A. Batalov, and V. Jacques, Proc. SPIE 7611, 761105 (2010).

${ }^{20}$ P. Neumann, J. Beck, M. Steiner, F. Rempp, H. Fedder, P. R. Hemmer, J. Wrachtrup, and F. Jelezko, Science 329, 542 (2010).

${ }^{21}$ F. Jelezko, T. Gaebel, I. Popa, M. Domhan, A. Gruber, and J. Wrachtrup, Phys. Rev. Lett. 93, 130501 (2004).

${ }^{22}$ T. van der Sar, Z. H. Wang, M. S. Blok, H. Bernien, T. H. Taminiau, D. M. Toyli, D. A. Lidar, D. D. Awschalom, R. Hanson, and V. V. Dobrovitski, Nature (London) 484, 82 (2012).

${ }^{23}$ R. Horodecki, P. Horodecki, M. Horodecki, and K. Horodecki, Rev. Mod. Phys. 81, 865 (2009).

${ }^{24}$ C. Boehme and D. R. McCamey, Science 336, 1239 (2012).

${ }^{25}$ W. K. Wootters, Phys. Rev. Lett. 802245 (1998).

${ }^{26}$ P. Neumann, N. Mizuochi, F. Rempp, P. Hemmer, H. Watanabe, S. Yamasaki, V. Jacques, T. Gaebel, F. Jelezko, and J. Wrachtrup, Science 320, 1326 (2008).

${ }^{27}$ T. Gaebel, M. Domhan, I. Popa, C. Wittmann, P. Neumann, F. Jelezko, J. R. Rabeau, N. Stavrias, A. D. Greentree, S. Prawer, J. Meijer, J. Twamley, P. R. Hemmer, and J. Wrachtrup, Nature Phys. 2, 408 (2006).

${ }^{28}$ G. Balasubramanian, P. Neumann, D. Twitchen, M. Markham, R. Kolesov, N. Mizuochi, J. Isoya, J. Achard, J. Beck, J. Tissler, V. Jacques, P. R. Hemmer, F. Jelezko, and J. Wrachtrup, Nature Mater. 8, 383 (2009). 